\title{
Cistoadenoma de glándulas salivales menores. Presentación de dos casos y revisión de la literatura
}

\section{Cystoadenoma of minor salivary glands. Two case reports and review of the literature}

\author{
De Castro Avellaner ME*, Figueiredo R**, Berini Aytés L***, Gay Escoda C****
}

\begin{abstract}
RESUMEN
Introducción: El cistoadenoma es un tumor salival benigno de origen epitelial muy infrecuente que se caracteriza por presentar múltiples proyecciones papilares y espacios microquísticos recubiertos de células cilíndricas o cuboideas. La OMS define el cistoadenoma como una neoplasia salival muy similar al tumor de Warthin, que carece de componente linfoide. La mayoría de cistoadenomas se han descrito a nivel de la laringe, la nasofaringe, así como en la glándula parótida y las glándulas lacrimales. Sin embargo, pueden localizarse con menor frecuencia en las mucosas labial y bucal, en la fosa tonsilar y en el paladar. El 35\% se sitúan en las glándulas salivales menores.

Casos clínicos: Se presentan dos casos clínicos diagnosticados histológicamente de cistoadenoma de una glándula salival menor, que acudieron al Servicio de Cirugía Bucal de la Clínica Odontológica de la Universidad de Barcelona. Se trata de dos varones de 79 y 86 años respectivamente, con antecedentes de diabetes no-insulinodependiente y úlcera gastroduodenal en el primer caso y de depresión en el segundo caso. Clínicamente, ambos pacientes presentaban una tumoración bien delimitada, de consistencia blanda, no ulcerada y sin adherencias a planos profundos. En el primer caso, la tumoración se situaba en la mucosa labial inferior, medía 1×0,7 cm, era de coloración idéntica a la mucosa adyacente y existía dolor a la palpación. En el segundo caso la tumoración era asintomática, de coloración azulada, se localizaba en la región del trígono retromolar derecho y medía $1,7 \times 1$ $\mathrm{cm}$. El tiempo de evolución para las lesiones fue de 3 y 2 meses respectivamente. El tratamiento en ambos casos fue la exéresis de la tumoración con márgenes de seguridad y el posterior examen anatomopatológico. Discusión: El cistoadenoma es una neoplasia salival benigna rara, descrita por primera vez por Skorpil en el año 1941, cuya definición y naturaleza ha sido controvertida. La clasificación de los tumores salivales de la OMS del año 1972 incluyó esta entidad dentro del apartado de adenomas monomorfos, sin embargo, en la última revisión del año 2005, el cistoadenoma constituye una variedad histológica independiente. En nuestra casuística, hemos encontrado 2 casos de cistoadenoma diagnosticados entre los años 1997 y 2006. Según la
\end{abstract}

* Odontóloga. Residente del Máster de Cirugía Bucal e Implantología Bucofacial. Facultad de Odontología de la Universidad de Barcelona. España.

* Odontólogo. Profesor asociado de Cirugía Bucal y Profesor del Máster de Cirugía Bucal e Implantología Bucofacial. Facultad de Odontología de la Universidad de Barcelona. Investigador del Instituto IDIBELL. Barcelona. España.

*** Médico Estomatólogo. Cirujano Maxilofacial. Profesor titular de Patología Quirúrgica Bucal y Maxilofacial y Profesor del Máster de Cirugía Bucal e Implantología Bucofacial. Decano de la Facultad de Odontología de la Universidad de Barcelona. Investigador del Instituto IDIBELL. Barcelona. España.

**** Médico Estomatólogo. Cirujano Maxilofacial. Catedrático de Patología Quirúrgica Bucal y Maxilofacial. Director del Máster de Cirugía Bucal e Implantología Bucofacial. Facultad de Odontología de la Universidad de Barcelona. Investigador coordinador del Instituto IDIBELL. Jefe del Servicio de Cirugía Bucal, Implantología Bucofacial y Cirugía Maxilofacial del Centro Médico Teknon. Barcelona. España. 
revisión de la literatura, el cistoadenoma constituye entre el 2 y el 4,7\% de todas las neoplasias de glándulas salivales menores. No existe acuerdo sobre la etiopatogenia del cistoadenoma, ya que hay autores que la consideran una verdadera neoplasia, mientras que otros autores creen que se trata de una hiperplasia del epitelio ductal glandular. Coincidiendo con los casos clínicos presentados, en la literatura se describe una predilección por el sexo masculino, siendo la edad de presentación más frecuente entre la $6^{\mathrm{a}}$ y la $8^{\mathrm{a}}$ décadas de la vida. El tratamiento de elección es la exéresis local, siendo infrecuente la recurrencia, siempre que la extirpación quirúrgica sea completa.

Palabras clave: Cistoadenoma papilar, adenoma, glándulas salivales menores.

\section{SUMMARY}

Introduction: Cystoadenomas are considered uncommon neoplasms of epithelial salivary origin and are characterized by having multiple papillary projections and microcystic spaces recovered by cuboid and cilindric cells. Cystoadenoma is defined by the WHO as a salivary neoplasia very similar to Warthin's tumour, but lacking lymphoid cells. The most common sites are the larynx, nasopharynx and lachrymal gland, although in some occasions these lesions can be found in the lips, buccal mucosa, palate and tonsillae. Thus $35 \%$ of cystoadenomas have minor salivary gland locations.

Case reports: Histological samples revealed 2 cases of minor salivary glands cystoadenomas in patients who attended the Oral Surgery department of the University of Barcelona. A man aged 79 years old with medical history of non insulin-dependent diabetes and gastric ulcer and another male patient with 86 years old with a previous history of depression. Macroscopic examination showed a well-limited tumour of tender consistency, mobile and without ulcerous surface. In the first case, the tumour was located in the lower lip, it measured $1 \mathrm{~cm}$ of length and $0.7 \mathrm{~cm}$ of width, had the same colour as the adjacent mucosa and was slightly painful when pressured. In the second case, the tumour was over the right retromolar area, and presented no symptoms and had a blue colour. The lesion measured $1.7 \mathrm{~cm}$ of length and $1 \mathrm{~cm}$ of width. The patients referred that the lesions were present for 2 to 3 months approximately. Surgical removal with margins was performed in both cases and the samples were sent for histological analysis.

Discussion: Cystoadenomas are uncommon salivary benign neoplasms described for the first time by Skorpil in 1941, and there has been some controversy about its origin and definition. WHO classification of salivary tumours in 1972 included this lesion as an adenoma. However in the latest review made in 2005, the cystoadenoma is considered a separate histological entity. We found two patients with these lesions diagnosed between 1997 and 2006. According to the literature, the frequency of cystoadenomas varies between $2 \%$ and $4.7 \%$ of all the minor salivary gland tumours. No etiopatogenic factors have been described and some authors consider this to be a real neoplasia, while others believe it is a ductal epithelium hyperplasia. Cystoadenomas are usually diagnosed between the 6th and the 8th decades of life. The treatment of choice is the local excision. No recurrences have been reported unless the lesion isn't fully removed.

Key words: Cystoadenoma, adenoma, minor salivary glands.

Fecha de recepción: 3 de diciembre de 2009.

Aceptado para publicación: 16 de diciembre de 2009 .

De Castro Avellaner ME, Figueiredo R, Berini Aytés L, Gay Escoda C. Cistoadenoma de glándulas salivales menores. Presentación de dos casos y revisión de la literatura. Av. Odontoestomatol 2011; 27 (3): 129-136.

\section{INTRODUCCIÓN}

El cistoadenoma de las glándulas salivales fue descrito por primera vez por Skorpil en 1941 (1) como un adenoma quístico en el que se pueden encontrar invaginaciones papilares epiteliales que se proyectan hacia la luz quística (2, 3). Esta definición se refiere a los cistoadenomas papilares, que fueron identificados primariamente (3). Posteriormente la Organización Mundial de la Salud (OMS) (4) incluyó una va- 
riante que se caracteriza por presentar una elevada cantidad de células mucosas; se constituyó así una nueva entidad; los cistoadenomas mucinosos. La última clasificación de la OMS conserva las subdivisiones y los engloba dentro de la categoría de los adenomas (5).

El cistoadenoma es un tumor muy similar al adenolinfoma (cistoadenoma papilar linfomatoso o tumor de Warthin) pero que carece de elementos linfoides. Las localizaciones más frecuentes son, según Chaudhry y cols. (6), la mucosa del paladar duro, la mucosa yugal, la porción posterior de la lengua (6) o el suelo de la boca (7). Existe gran variabilidad en cuanto a la incidencia de esta entidad (desde el $2 \%$ hasta el $4,7 \%$ de todos los tumores de glándulas salivales menores) (8).

Debido a esta frecuencia tan baja no existen estudios con una muestra amplia, por que creemos que la presentación de dos casos clínicos de cistoadenomas en las glándulas salivales menores puede ser interesante.

Las características que se describen a continuación, ayudarán a establecer un diagnóstico diferencial correcto con otras entidades que presentan características clínicas similares para implementar de esta forma el tratamiento adecuado.

\section{CASOS CLÍNICOS}

\section{Caso 1}

Hombre, de 79 años de edad, que acudió al Servicio de Cirugía Bucal de la Clínica Odontológica de la Universidad de Barcelona, remitido desde el Centro de Atención Primaria para la valoración de una tumoración ubicada en la mucosa yugal izquierda que presentaba una evolución de 3 meses.

El paciente era fumador de 20 cigarrillos diarios y con más de 30 años de duración del hábito. Como antecedentes personales refería diabetes no insulinodependiente, artrosis, úlcera gastroduodenal no activa, hipercolesterolemia y había sido intervenido quirúrgicamente por opacidad en el cristalino 4 años antes de la consulta en nuestro Servicio.
En la exploración bucodental se podía observar un edentulismo total del maxilar superior y un edentulismo parcial en la mandíbula (únicamente conservaba el 4.3 y el 4.4 con periodontitis crónica).

En el examen clínico de los tejidos blandos se podía apreciar una masa sobreelevada que medía aproximadamente $2 \mathrm{~cm}$ de largo por $1 \mathrm{~cm}$ de ancho, localizada entre la cresta alveolar del tercer cuadrante y el labio inferior, sin sobrepasar la línea media (Fig. 1A).

La consistencia era blanda, nodular y la palpación provocaba un drenaje de líquido seroso por orificios de $1 \mathrm{~mm}$ de diámetro presentes en la superficie de la tumoración. La mucosa bucal que cubría la tumoración presentaba un color homogéneo con un fondo ligeramente azulado.

En la ortopantomografía se podía apreciar la inclusión del 1.3 que no había ocasionado sintomatología. A parte de este hallazgo, no se encontraron alteraciones en la exploración radiológica. Tampoco se palpaban adenopatías o masas cervicales.

En los controles efectuados previamente a la toma de biopsia, la tumefacción experimentó cambios de tamaño desde un abultamiento de $2 \mathrm{~cm}$ hasta la regresión casi completa. El principal diagnóstico de presunción fue el de linfangioma.

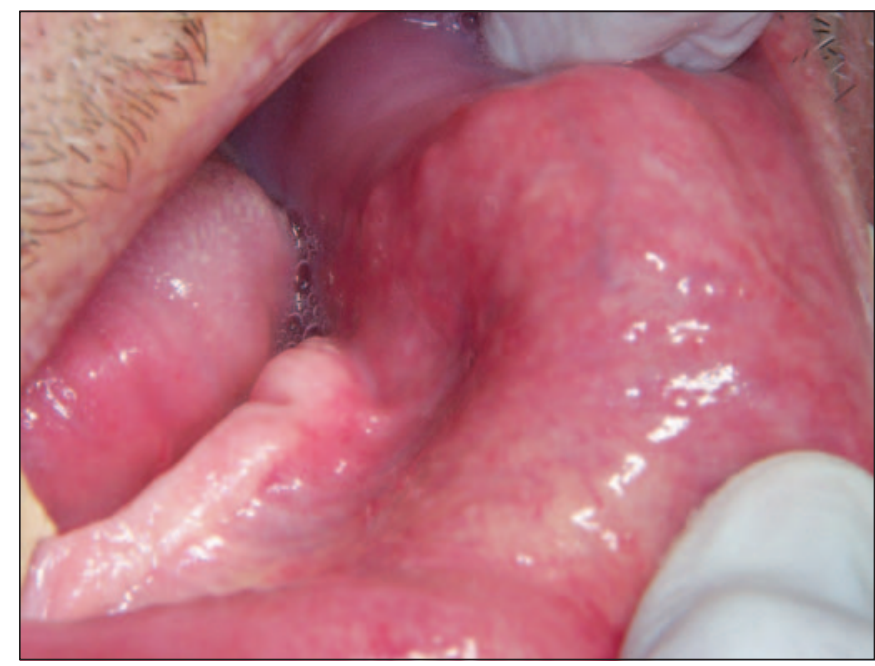

Fig. 1A. Caso 1. Aspecto de la tumoración al evertir el labio inferior. 
Tras efectuar una biopsia incisional se practicó el análisis anatomopatológico cuyo resultado fue de cistoadenoma papilar de glándula salival menor (Fig. 1B). Dos semanas después, se hizo la resección completa de la tumoración bajo anestesia local sin complicaciones intraoperatorias (Fig. 1C). El análisis de la pieza operatoria resecada confirmó el diagnóstico previo.

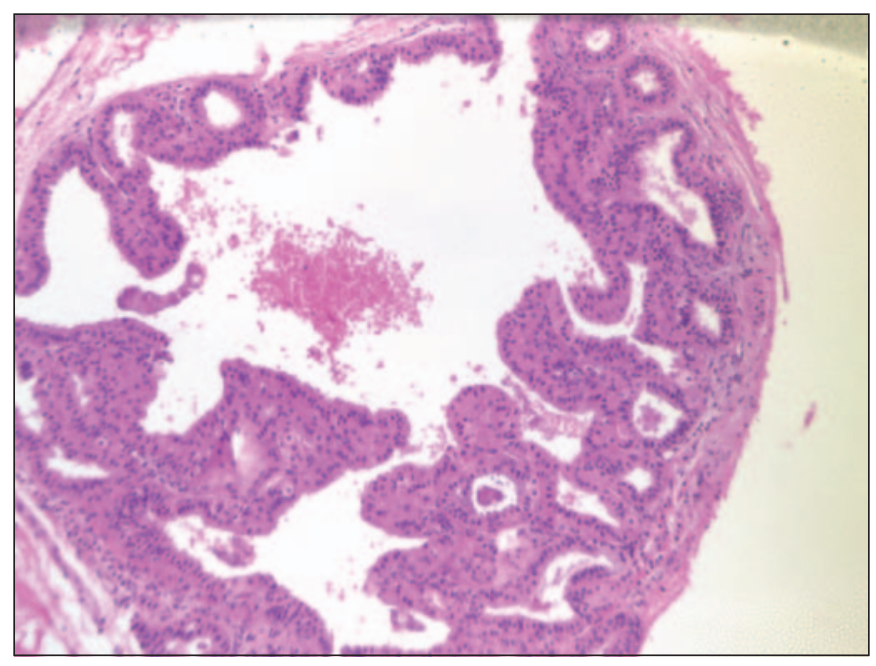

Fig. 1B. Caso 1. Muestra teñida con hematoxilina eosina (x20). Se observan las proyecciones papilares que se dirigen hacia la luz quística.

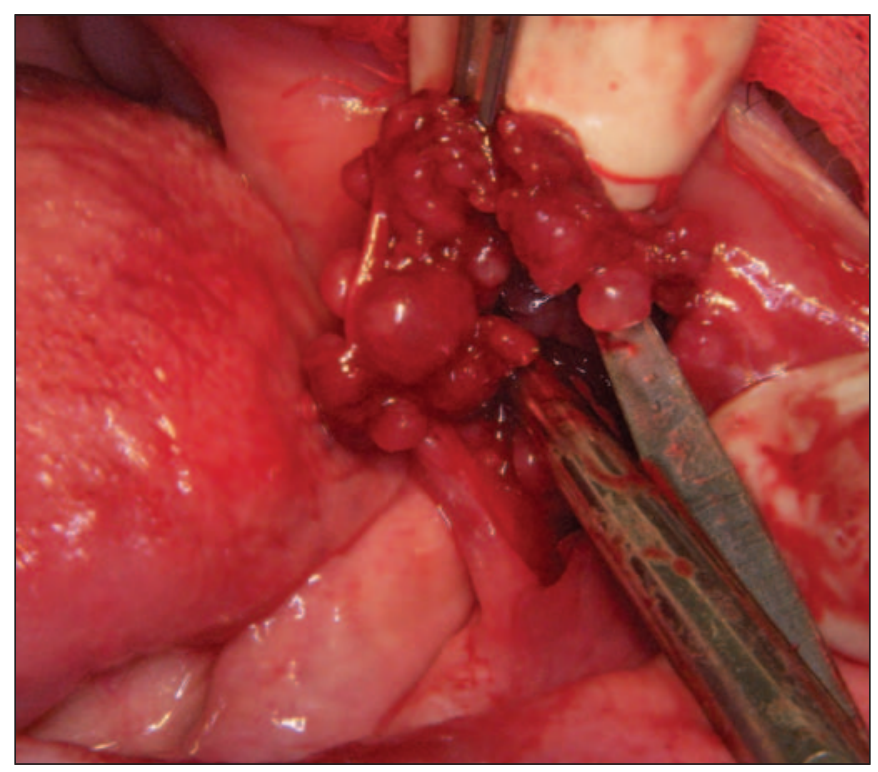

Fig. 1C. Caso 1. Aspecto de la lesión durante la disección-exéresis con tijeras de Metzembaum.
Como secuela de la intervención quirúrgica apareció un área de hipoestesia en el labio inferior. Transcurrido un año y tres meses, se recuperó por completo la sensibilidad de esta zona. No se observó recurrencia de la tumoración en el control anual.

\section{Caso 2}

Hombre de 86 años de edad, remitido al Servicio de Cirugía Bucal de la Clínica Odontológica de la Universidad de Barcelona por presentar una lesión de aspecto azulado en el trígono retromolar.

El paciente padecía Parkinson, depresión, hernia de hiato e hipertensión.

Para el tratamiento de estas entidades el paciente tomaba Alfuzosina (Benestan retard ${ }^{\circledR}$, Sanofi Aventis, Barcelona, España), Carbidopa-levodopa (Sinemet ${ }^{\circledR}$, Bristol-Myers Squibb, Barcelona, España), Olanzapina (Zyprexa ${ }^{\circledR}$, Lilly y Dista, Madrid, España), Escitalopram (Cipralex ${ }^{\circledR}$, Lunbeck España, Barcelona, España), Omeprazol (Omeprazol Sandoz EFG, Sandoz Farmacéutica, Barcelona, España), Pantoprazol sódico (Pantecta ${ }^{\circledR}$, Nycomed Pharma, Madrid, España). Además refería hipersensibilidad a la penicilina.

En la exploración bucodental, se podía apreciar una lesión sobreelevada de color azulado en el trígono retromolar derecho de consistencia fibrosa a la palpación y que no provocaba dolor (Fig. 2A). En la ortopantomografía se podían observar los restos radiculares de 3.3, 3.2. 3.1 y 4.3.

Basándose en la clínica y en las características de la lesión, se estableció el diagnostico de presunción de angioma. Ya que este era el principal diagnostico de sospecha, se decidió hacer la exéresis mediante el láser de $\mathrm{CO}_{2}$ intentando minimizar de esta forma la hemorragia que pudiera producirse durante la resección de la tumoración (Fig. 2B).

No se produjeron complicaciones intra o postoperatorias durante la exéresis y se remitió el tejido extraído para el estudio histológico. El resultado del análisis anatomopatológico de la lesión fue de cistoadenoma papilar (Fig. 2C). Durante el año posterior a la exéresis no apareció recurrencia de la lesión. 


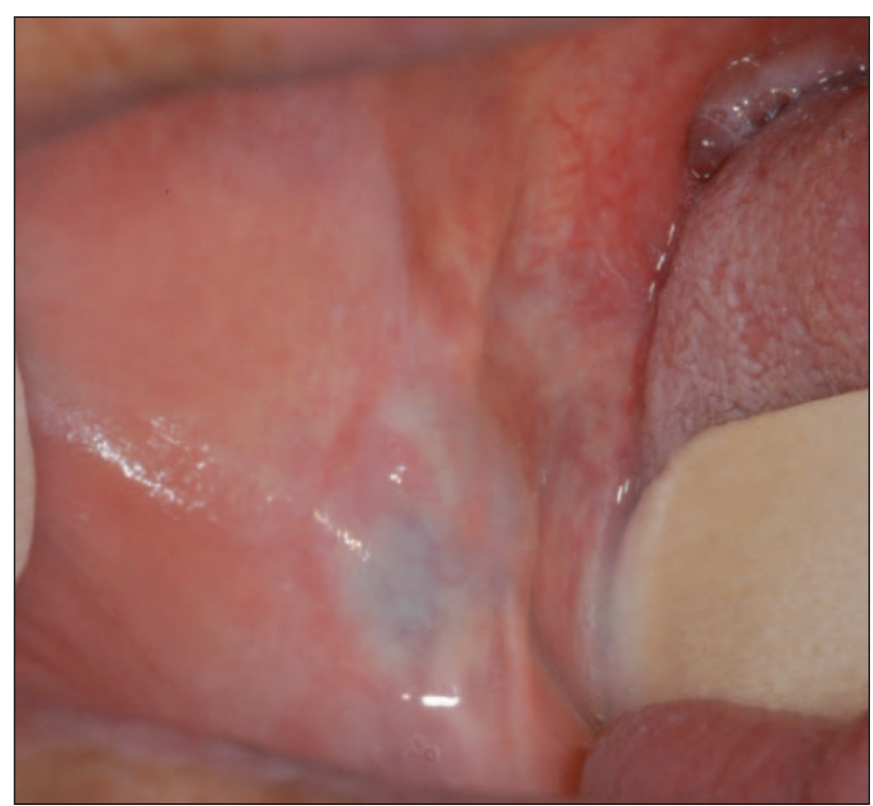

Fig. 2A. Caso 2. Lesión de aspecto azulado localizada en el trígono retromolar derecho.

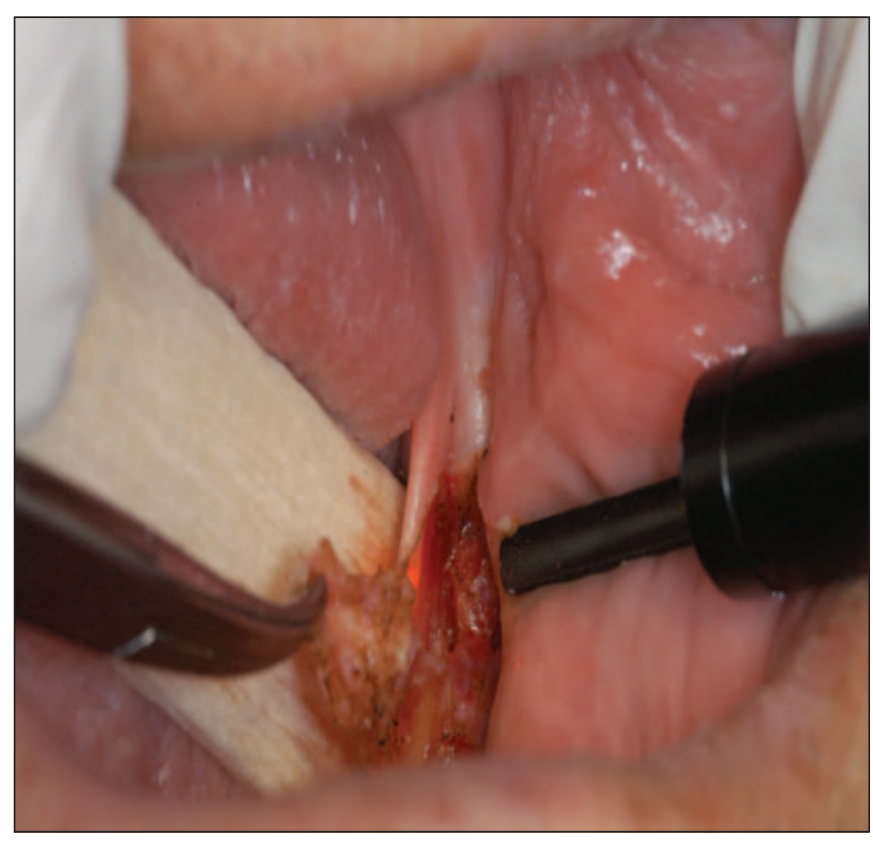

Fig. 2B. Caso 2. Exéresis-biopsia de la lesión localizada en el trígono retromolar mediante el láser de $\mathrm{CO}_{2}$.

\section{DISCUSIÓN}

En la primera clasificación publicada por la OMS en 1972 (3) se describió el cistoadenoma papilar como

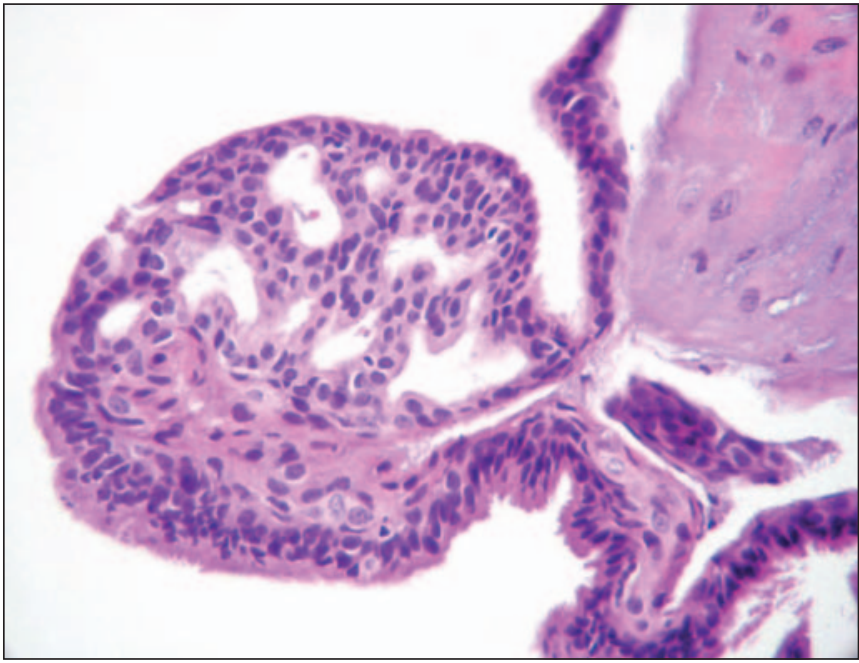

Fig. 2C. Caso 2. Detalle del área papilar de la lesión. La papila se encuentra rodeada de un epitelio columnar pseudoestratificado ciliado. Existe infiltración de células inflamatorias en el tejido conjuntivo, en el que predominan las células plasmáticas.

una entidad independiente, separada del cistoadenoma papilar oncocítico o Tumor de Warthin debido a la ausencia de células linfoides, característica principal que diferencia ambos tumores. Se incluía al cistoadenoma dentro del grupo de los adenomas monomorfos en base al patrón histológico del tumor.

En 1978, Kerpel y cols. (9) publicaron un caso de un cistoadenoma en la mucosa bucal, proponiendo como diagnóstico provisional el de mucocele. Tras el análisis histológico, encontraron la imagen de un cistoadenoma papilar, pero compuesto en este caso por grupos de células mucosas sobre la membrana basal.

Unos años más tarde, esta clasificación fue modificada por Seifert y Sobin (4) y se publicó en 1991. En este momento, el cistoadenoma pasó a constituir una entidad histopatológica independiente que, además, se subdividía en los tipos papilar y mucinoso.

Desde el 2005 (5) se incluye al cistoadenoma dentro de los tumores epiteliales benignos en el grupo de los adenomas monomorfos conservando las subdivisiones antes comentadas (Tabla 1).

La etiopatogenia de esta tumoración es controvertida y algunos autores como Chaudhry y cols. (10) 


\section{TABLA 1.- CLASIFICACIÓN DE LA OMS DE LOS TUMORES BENIGNOS DE ORIGEN EPITELIAL DE LAS GLÁNDULAS SALIVALES (5)}

\section{Tumores benignos epiteliales}

Adenoma pleomorfo.

Mioepitelioma.

Adenoma de células basales.

Tumor de Warthin.

Oncocitoma.

Adenoma canalicular.

Adenoma sebáceo.

Linfadenoma: sebáceo, no sebáceo.

Papilomas ductales: papiloma ductal invertido, papiloma intraductal y sialadenoma papilífero.

Cistoadenoma: mucinoso y papilar.

sugieren que el cistoadenoma papilar podría tratarse de una reacción hiperplásica papilar intraductal, mientras que otros investigadores propugnan que esta entidad sería una variante quística de los adenomas monomorfos.

Bauer y Bauer (11) atribuyen el origen de esta tumoración al epitelio indiferenciado de los ductus intercalares de la glándula salival y otros como Kerpel y cols. (9) no lo consideran un verdadero tumor sino una hiperplasia de este epitelio del conducto salival.

Normalmente, el epitelio que rodea las cavidades quísticas en el cistoadenoma está libre de células linfoides y presenta una notable diferenciación de las células escamosas. Se puede observar infiltrado de células inflamatorias aunque no muy abundante en el estroma del área subyacente a las células de los ductus de las proyecciones papilares.

La frecuencia de presentación de esta entidad es muy controvertida en la literatura. En este sentido, la mayor parte de los autores coinciden en que su in- cidencia es baja. Mientras que Waldron y cols. (7) reportan un $8,1 \%$ de cistoadenomas dentro del total de los tumores benignos, otros como Chaudhry y cols. (6) cifraron en 1961 en un 2\% la frecuencia de estas lesiones. La frecuencia descrita por estos últimos, podría considerarse como una de las referencias más fiables, ya que la cantidad de la muestra estudiada sigue siendo hasta el momento la más amplia (1.414 casos de tumores de glándulas salivales menores). Sin embargo, los criterios diagnósticos y la nomenclatura que fueron establecidos en 1961 son diferentes de los actuales; lo que implica que debemos actuar con cautela a la hora de interpretar estos datos.

Por este motivo, en un intento de actualizar estos datos y de describir la frecuencia individual de cada tumor, Buchner y cols. (12) publicaron un trabajo en el que revisan la frecuencia relativa de los tumores intrabucales de glándulas salivales menores en la población del norte de California. Este estudio basándose en la última clasificación de la OMS, obtienen una incidencia del 6,3\% más cercana a la descrita por Waldron y cols. (7) en 1988.

Más recientemente, Wang y cols. (13) revisaron 737 tumores de glándulas salivales menores, encontrando únicamente 6 cistoadenomas de entre los casos estudiados, por lo que la incidencia que reportan estos autores estaría muy por debajo de la que estipulaban los estudios previos.

Se han publicado algunos casos $(1,2,14-16)$ de cistoadenomas de glándulas salivales menores pero no hay acuerdo sobre la localización preferente de esta tumoración.

En el diagnóstico diferencial se podrían incluir lesiones como los quistes de retención mucosa o los mucoceles, los adenomas pleomorfos, la queilitis glandular y los linfangiomas. Los quistes de retención mucosa no presentan predilección por el sexo. Presentan variaciones en el tamaño de la lesión al igual que uno de nuestros casos y suelen ubicarse preferentemente en el labio inferior. Si bien los dos casos tenían un tiempo de evolución de 2-3 meses, no referían antecedentes de traumatismos y más concretamente en el caso en que la tumoración apareció en el trígono retromolar (caso 2), es poco fre- 
cuente que un quiste de retención mucosa aparezca en esta zona.

La localización más frecuente del adenoma pleomorfo es el paladar, lo cual difiere de las dos lesiones que presentaban nuestros pacientes. Representa el $50 \%$ de los tumores de glándulas salivales menores y a pesar de que el aspecto clínico puede ser similar, el período de evolución del adenoma pleomorfo suele ser más lento y progresivo que el de las lesiones documentadas en este artículo.

Los linfangiomas son tumoraciones que acostumbran a observarse en el dorso de la lengua y en los labios, presentando variaciones de tamaño durante su curso, al igual que en el caso 1 .

El diagnóstico de sospecha en el segundo caso se dirigió hacia el hemangioma, debido a la coloración azulada que presentaba, el curso clínico y la localización de la tumoración.

Se describe un caso en la literatura (16) con características clínicas similares al caso 1 que presentamos. La lesión, localizada en el labio superior, drenaba líquido seropurulento por orificios de pequeño diámetro que se observaban en la superficie de la tumoración. En el estudio histológico del caso descrito por Stoopler y cols. (17) se encuadró como un cistoadenoma papilar oncocítico, pero tras la exéresis completa, el diagnóstico cambió a queilitis glandular. Se encontraron zonas de hiperplasia y metaplasia escamosa con adelgazamiento del epitelio en el análisis de la pieza de resección. En nuestro caso no se encontraron estas alteraciones del epitelio tanto en la biopsia como en el estudio histológico tras la eliminación de la tumoración.

El tratamiento del cistoadenoma se basa en la exéresis completa de la lesión eliminando el tejido patológico sin necesidad de dejar márgenes de seguridad (12-18).

Por el momento no se ha publicado ningún caso de recurrencia de las lesiones durante un período de seguimiento mínimo de 2 años y nosotros tampoco hemos registrado recidivas. Aunque algunos autores consideran que los cistoadenomas son tumores potencialmente malignos, no hemos encontrado referencias de malignizaciones en la literatura (12-18).

\section{AGRADECIMIENTOS}

Este estudio ha sido realizado por el grupo de investigación consolidado "Patología y Terapéutica Odontológica y Maxilofacial” del Instituto de Investigación Biomédica de Bellvitge (IDIBELL), con el soporte económico del convenio docente-asistencial de Cirugía Bucal entre la Universidad de Barcelona, el Consorcio Sanitario Integral y el Servicio Catalán de la Salud de la Generalitat de Catalunya.

\section{BIBLIOGRAFÍA}

1. Akin RK, Kreller AJ, Walters PJ. Papillary cystadenoma of the lower lip -report of a case. J Oral Surg 1973;31:858-60.

2. Alexis J, Dewbrow V. Papillary cystadenoma of a minor salivary gland. J Oral Maxillofac Surg 1995; 53:70-2.

3. Thackray AC, Sobin LH. Histologic typing of salivary gland tumors. Geneva: World Health Organization; 1972.

4. Seifert G, Sobin LH. World Health Organization international histological classification of tumours: histological typing of salivary gland tumours. 2nd ed. Berlin, Germany: Springer-Verlag; 1991.

5. Barnes EL. World Health Organization Classification of Tumours: Pathology and Genetics of Head and Neck Tumours. 1st ed. Lyon, France: IARC Press; 2005.

6. Chaudhry AP, Vickers RA, Gorlin RJ. Intraoral minor salivary gland tumours, an analysis of 1414 cases. Oral Surg Oral Med Oral Pathol 1961;14:1194-226.

7. Waldron CA, El-Mofty SK, Gnepp DR. Tumors of the intraoral minor salivary glands: a demographic and histologic study of 426 cases. Oral Surg Oral Med Oral Pathol 1988;66:323-33.

8. Guccion J, Redman R, Calhoun N, Saini N. Papillary cystadenoma of the palate: a case report and ultrastructural study. J Oral Maxillofac Surg 1997;55:759-64. 
9. Kerpel SM, Freedman PD, Lumerman H. The papillary cystadenoma of minor salivary gland origin. Oral Surg 1978;46:820-6.

10. Chaudhry AP, Labay GR, Yamane GM, Jacobs MS, Cutter LS, Watkins KV. Clinicopathologic and histogenetic study of 189 intraoral minor salivary gland tumors. J Oral Med 1984;39:58-78.

11. Bauer WH, JD Bauer. Classification of glandular tumors of salivary glands; study of 143 cases. Arch Pathol 1958;55:328-46.

12. Buchner A, Merrell PW, Carpenter WM. Relative frequency of intra-oral minor salivary gland tumors: a study of 380 cases from northern California and comparison to reports from other parts of the world. J Oral Pathol Med 2007;36:207-14.

13. Wong D, Li Y, He H, Liu L, Wu L, He Z. Intraoral minor salivary gland tumors in a Chineses population: A retrospective study on 737 cases. Oral Surg Oral Med Oral Pathol Oral Radiol Endod 2007;104:94-100.

14. Matsuzaka K, Kokubu E, Takeda E, Tanaka Y, Shimono M, Inoue T. Papillary cystadenoma arising from the upper lip: a case report. Bull Tokyo Dent 2003;44:213-6.
15. Tsurimi K, Kamiya H, Yokoi M, Kameyama Y. Papillary oncocytic cystadenoma of palatal minor salivary gland: a case report. J Oral Maxillofac Surg 2003;61:631-3.

16. Cathoun NR, Cerine FC, Mathews MJ. Papillary cystadenoma of the upper lip. Oral Surg 1966; 21:782-5.

17. Stoopler E, Carrasco L, Stanton D, Pringle G, Sollecito T. Cheilitis glandularis: An unusual histopathologic presentation. Oral Surg Oral Med Oral Pathol Oral Radiol Endod 2003;95: 312-7.

18. Matsuzaka K, Kokubu E, Takeda E, Tanaka Y, Shimono M, Inoue T. Papillary cystadenoma arising from the upper lip: A case report. Bull Tokyo Dent 2003; 44: 213-6.

\section{CORRESPONDENCIA}

Dr. Cosme Gay-Escoda

Centro Médico Teknon

Instituto de Investigación IDIBELL

Vilana, 12

08022 Barcelona. España

E-mail: cgay@ub.edu

Página web: http://www.gayescoda.com 